\title{
Electron correlations and bond-length fluctuations in copper oxide superconductors: electron versus hole doping
}

\author{
L. Hozoi ${ }^{1}$ and S. Nishimoto ${ }^{2}$ \\ ${ }^{1}$ Max-Planck-Institut für Festkörperforschung, Heisenbergstrasse 1, 70569 Stuttgart, Germany \\ ${ }^{2}$ Max-Planck-Institut für Physik Complexer Systeme, Nöthnitzer Str. 38, 01187 Dresden, Germany
}

(Dated: November 16, 2018)

\begin{abstract}
We investigate the nature of the electronic ground state and electron-lattice couplings for doped chains of $\mathrm{CuO}_{4}$ plaquettes or $\mathrm{CuO}_{6}$ octahedra. The undoped configuration implies here $\mathrm{Cu} 3 d^{9}$ and $\mathrm{O} 2 p^{6}$ formal valence states. The results of multiconfiguration calculations on 4-plaquette (or 4-octahedra) linear clusters indicate strong electron-lattice interactions and polaronic behavior of the doped particles, for both electron and hole doping. For certain phases of the oxygen-ion halfbreathing distortions a multi-well energy landscape is predicted. Since each well is associated to carriers localized at different sites, the half-breathing displacements induce charge transfer along the chain. In the case of hole-doping, the trends found by ab initio multiconfiguration calculations on 4-octahedra clusters are confirmed by density-matrix renormalization-group calculations for a $p-d$, extended Hubbard model with chains of few tens of $\mathrm{CuO}_{4}$ plaquettes. Under the assumption of charge separation and the formation of 1/3-doped stripes, our results seem to support the traveling charge-density wave scenario proposed in some recent contributions for superconductivity in copper oxides.
\end{abstract}

\section{INTRODUCTION}

A remarkable property of the copper oxide compounds is that superconductivity can be obtained in these systems by both hole and electron doping. Holes added to the insulating, antiferromagnetic (AFM) $\mathrm{CuO}_{2}$ network have $\mathrm{O} 2 p$ character, see for example [1, 2, [3, 4, [5, 6]. Results of recent $a b$ initio, multiconfiguration calculations on clusters including several $\mathrm{CuO}_{6}$ octahedra also indicate strong electron-lattice couplings [6]. It was found in Ref. [6] that the lowest energy configurations imply Zhang-Rice (ZR) 7] like, quasi-localized states on distorted $\mathrm{CuO}_{4}$ plaquettes. However, O-atom halfbreathing displacements that restore the high-symmetry structure with identical $\mathrm{Cu}-\mathrm{O}$ distances induce strong charge redistribution. The $\mathrm{O} 2 p$ hole can be (partially) transferred onto a single anion to give an electronic wavefunction with a dominant contribution from a ...- $\mathrm{Cu} d^{9}-$ $\mathrm{O} p^{5}-\mathrm{Cu} d^{9}-\ldots$ configuration. The ZR like singlet can hop thus within the $\mathrm{CuO}_{2}$ plane via such $d^{9}-p^{5}-d^{9}$ states through coupling to the oxygen vibrations. According to the results from [6], the energy barrier associated with this double-well potential is $300-400 \mathrm{meV}$ for an isolated O $2 p$ hole.

For not too high doping levels, these ZR type singlets are sufficiently far apart such that they do not overlap. It seems that at the optimal hole concentration $p \approx 1 / 6$ and low temperatures, spin correlations and hole-hole Coulomb interactions determine within the $\mathrm{CuO}_{2}$ plane an unique, charge inhomogeneous arrangement of the $\mathrm{O}$ $2 p$ holes, see for example [8, 9, 10, 11, 12. J. B. Goodenough [12] argued that at $p \approx 1 / 6$ the doped holes form alternating, hole-free and $1 / 3$-doped stripes of $\mathrm{CuO}_{4}$ plaquettes. Using the $a b$ initio results from Ref. [6] for holedoped clusters, the charge and spin distribution for a $1 / 3$-doped stripe could be schematically represented as in Fig. 1(a). The squares correspond to $\mathrm{ZR}$ states on $\mathrm{CuO}_{4}$ plaquettes with shorter $\mathrm{Cu}-\mathrm{O}$ bonds along the chain $[\underline{6}$. In the undistorted structure, a quasilocalized O $2 p$ hole favors ferromagnetic couplings between adjacent $\mathrm{Cu}$ ions, as initially predicted in 13, 14, 15]. A three-site, $d^{9}-p^{5}$ $d^{9}$ spin-polaron model was used by Kochelaev et al. to explain the electron paramagnetic resonance (EPR) signal at different doping levels in $\mathrm{La}_{2-x} \mathrm{Sr}_{x} \mathrm{CuO}_{4}$ [16].

We speculated in [6] that for a distribution of the oxygen holes as that shown in Fig. 1(a), the strong coupling between the nuclear and electronic motions, the hole-hole Coulomb repulsion, and the in-plane spin interactions would cause strong correlations among half-breathing Oatom vibrations on every third, $\mathrm{ZR}$ like $\mathrm{CuO}_{4}$ plaquette along the doped chains. The superconductivity might

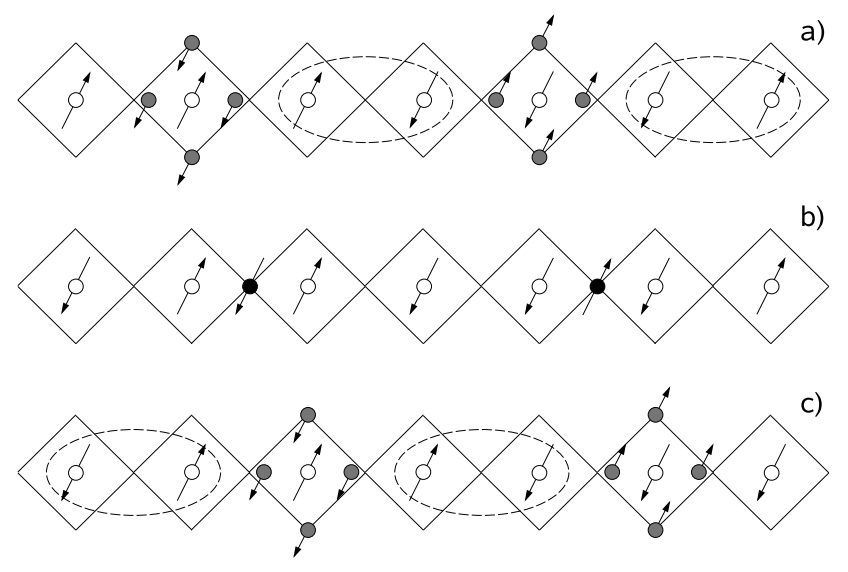

FIG. 1: Possible representation of the traveling chargedensity/spin-density wave along a $1 / 3$ hole-doped $\mathrm{CuO}_{4}-$ $\mathrm{CuO}_{4}-\ldots$ chain, see Section III and Ref. 6]. The O $2 p$ holes are shown schematically with black or gray color. The ellipses indicate spin-singlet couplings. 
be related to the collective, coherent motion of the $\mathrm{O}$ $2 p$ holes along the $1 / 3$-doped chains, which is associated with phase coherence among the kind of O-atom vibrations shown in Fig. 1(a-c). This evokes the vibronic mechanism and the traveling charge-density/spin-density wave $(\mathrm{CDW} / \mathrm{SDW})$ scenario suggested by Goodenough 12].

Spatially localized bound states of two electrons were analyzed within a two-dimensional Holstein-Hubbard model on a square lattice by Proville and Aubry [17]. Two electrons bound into a localized singlet state as a result of strong electron-lattice interactions are often referred to as a bipolaron. It was found in Ref. [17] that for certain values of the model parameters a "quadrisinglet" (QS) state similar to the ZR like state discussed above can be formed. The near-degeneracy between the QS "bipolaron" and a two-site spin-singlet bipolaron can lead to a sharp reduction of the bipolaron effective mass. Proville and Aubry argued that such near-degeneracy effects plus quantum tunneling effects associated with a rather flat energy landscape should be crucial ingredients in the theory of "bipolaronic" superconductivity [18].

The fact that the $a b$ initio calculations from Ref. 6 ] confirm a number of distinct effects and concepts (that is, $\mathrm{ZR}$ type states on $\mathrm{CuO}_{4}$ plaquettes [7], Kondo like interactions and ferromagnetic couplings between $3 d_{x^{2}-\nu^{2}}$ electrons adjacent to a quasilocalized oxygen hole 13, 14, 15], near-degeneracy between the QS configuration and a two-site spin-singlet configuration and strong electron-
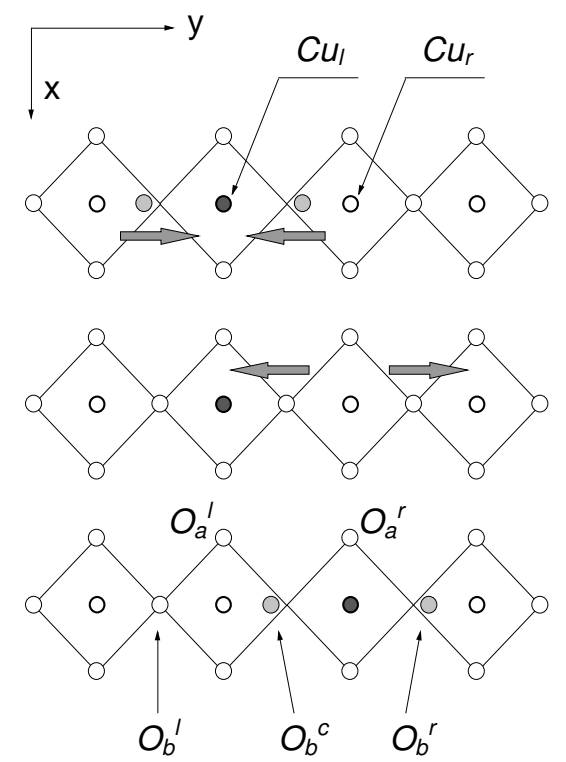

FIG. 2: Electron-doped, 4-plaquette cluster and the phases of the O-ion half-breathing distortions investigated by CAS SCF calculations. A Cu $3 d_{x^{2}-y^{2}}^{2}$ ion is shown with a filled, darkgray circle. From left to right, the transition metal sites are labeled as $\mathrm{Cu}_{l l}, \mathrm{Cu}_{l}, \mathrm{Cu}_{r}$, and $\mathrm{Cu}_{r r}$. Ligands that are displaced from the middle positions are shown with light-gray color. lattice couplings [17]) deduced or proposed on the basis of model Hamiltonians that rest on quite different approximations and describe rather different types of interactions is very interesting. It illustrates both the power of and the need for first principles, explicitly correlated methods in the study of these materials. Such calculations on not very large clusters are sometimes essential for understanding the "local" many-body physics and for setting up the "right" model for the extended system, as recently shown in the case of a mixed-valence vanadium oxide, $\mathrm{NaV}_{2} \mathrm{O}_{5}$ [19]. Near-degeneracy and pseudo JahnTeller effects involving O-hole $d^{1}-p^{5}-d^{1}$ configurations similar to the $d^{9}-p^{5}-d^{9}$ three-site spin-polaron in the doped copper oxides appear to be the source of most of the intriguing properties of this compound.

In the present paper we report results of $a b$ initio wave-function based, embedded cluster calculations for the electron-doped case. We investigate the ground-state electron distribution and electron-lattice couplings. For an 1-doped-electron, 4-plaquette linear cluster, our data show that the lowest energy state corresponds to a configuration where the extra electron is localized on a $\mathrm{CuO}_{4}$ plaquette with elongated $\mathrm{Cu}-\mathrm{O}$ bonds. As in the holedoped system, the O-atom half-breathing displacements interact with the electronic degrees of freedom and may induce charge transfer along the $\mathrm{CuO}$ chain. We shall describe various aspects of this kind of interactions and discuss the similarities and differences with the hole-doped situation.

\section{1-DOPED-ELECTRON, 4-PLAQUETTE LINEAR CLUSTERS: BOND-LENGTH FLUCTUATIONS AND INTER-SITE CHARGE TRANSFER}

Multiconfiguration, complete active space (CAS) selfconsistent-field (SCF) electronic structure calculations were carried out on linear clusters including four $\mathrm{CuO}_{4}$ plaquettes. The CASSCF method is designed to provide an unbiased description of systems where neardegeneracy occurs among several electron configurations [20]. It is based on a partitioning of the total orbital space into three subsets, inactive, active, and virtual orbitals. The inactive orbitals are doubly occupied in all configurations and should be chosen as the orbitals that are not expected to contribute to near-degeneracy correlation effects. The active orbitals are subject to no restriction on their occupation numbers and the virtual orbitals are unoccupied in all configurations. The CASSCF wavefunction is constructed as a full configuration-interaction (CI) expansion in the configurational space spanned by the active orbitals. Since both the CI coefficients and the orbitals are variationally optimized, the CAS SCF wavefunction is extremely flexible. It is this flexibility that makes the CAS SCF method well suited for the description of competing valence structures, bond breakings, and dissociation processes. 
We used the crystal parameters reported for the electron-doped copper oxide superconductor compound $\mathrm{Sr}_{1-y} \mathrm{Nd}_{y} \mathrm{CuO}_{2}$ 21], with $y \approx 0.16, \mathrm{Cu}-\mathrm{O}$ distances of $1.97 \AA$, and no apical oxygens. The 4-plaquette cluster was embedded in an array of point charges that reproduce the Madelung potential associated with an idealized $\mathrm{SrCuO}_{2}$ ionic system. We imposed $C_{2 v}$ symmetry restrictions, with $x y$ and $y z$ mirror planes (see Fig.2), and employed the following gaussian-type, atomic natural orbital basis sets for the $\left[\mathrm{Cu}_{4} \mathrm{O}_{13}\right]$ cluster: $\mathrm{Cu}$ $21 s 15 p 10 d 6 f / 5 s 4 p 3 d 1 f$ and $\mathrm{O} 14 s 9 p 4 d / 4 s 3 p 1 d$ [22]. It is known by now that such basis sets provide a quite accurate description of both ground-state and excited-state properties in a large variety of transition metal (TM) oxides, see for example [5, 19, 23, 24] and 25]. The $\mathrm{Cu}$ and $\mathrm{Sr}$ neighboring ions were represented by total ion potentials 26]. The calculations were performed with the MOLCAS program package [27].

With one electron added to the undoped, formally $\mathrm{Cu} d^{9} \mathrm{O} p^{6}$ configuration, the minimal active orbital space should consist of four (or even three) open-shell $3 d_{x^{2}-y^{2}}$ orbitals. However, trial CAS SCF calculations were carried out with few virtual orbitals added to the active space. It turned out that these extra orbitals have either $\mathrm{Cu} 4 s$ or $\mathrm{Cu} 3 d^{\prime}$ character, where $3 d^{\prime}$ is a more diffuse orbital than $3 d$ [28], and non-negligible occupation numbers. Also, large effects were observed on the absolute energies, as compared to the minimal active space.

The relevance of the $4 s$ orbital to the local-density approximation (LDA) conduction bands and to the inplane/inter-plane hoppings was analyzed for several copper oxide compounds in Refs. [29, 30]. A clear correlation was observed [30] between the value of the critical temperature $T_{c}$ and the energy and specific composition of the so-called axial orbital, a hybrid with mixed $\mathrm{Cu} 4 s$, $\mathrm{Cu} 3 d_{3 z^{2}-r^{2}}$, and apical-oxygen $2 p_{z}$ character. The importance of the $\mathrm{Cu} 4 s$ and $4 p$ orbitals for an accurate description of the electron occupation of the $\mathrm{Cu} 3 d_{x^{2}-y^{2}}$ and $\mathrm{O} 2 p_{x} / 2 p_{y}$ orbitals in model Hamiltonian calculations and also their role in screening the on-site $\mathrm{Cu} 3 d$ Coulomb repulsion was discussed in [2].

We investigated the dependence of the cluster total energy on various distortions and varied the positions of the $\mathrm{Cu}$ ions and of those oxygens $\left(\mathrm{O}_{b}\right.$, see Fig.2) bridging the cations. For such a cluster, energetic effects associated with displacements of the $\mathrm{O}_{a}$ oxygen ions, see Fig.2, are not meaningful, because part of the nearest neighbors of these ligands are modeled by effective potentials or even point charges. Nevertheless, under the assumption of charge segregation into filamentary stripe segments and one-dimensional conduction [11, 12, 13], the distortions along the $y$ axis should be the most relevant. We found that the cluster energy is minimized for a geometry where the $\mathrm{Cu}-\mathrm{O}_{b}$ bonds on one of the $\mathrm{CuO}_{4}$ plaquettes are stretched by approximately $8 \%$ and the nearest $\mathrm{Cu}$ neighbors are also displaced by about $1 \%$ of the initial $\mathrm{Cu}-\mathrm{O}$ bond length, each of them in the same direction as the adjacent oxygen. The doped electron is accommodated in the $3 d_{x^{2}-y^{2}}$ orbital on the plaquette with elongated $\mathrm{Cu}-\mathrm{O}_{b}$ bonds to give a $\mathrm{Cu} 3 d_{x^{2}-y^{2}}^{2}$ configuration. We report results for geometries where either the $\mathrm{Cu}_{l}-\mathrm{O}_{b}$ or $\mathrm{Cu}_{r}-\mathrm{O}_{b}$ bonds are stretched, see Fig.2, and discuss the changes in the charge distribution for these two plaquettes and the variation of the cluster energy. However, in this study we keep all $\mathrm{Cu}$ ions at fixed positions. This has only minor effects on the ground-state wave-function and energy. Our active space includes four $3 d_{x^{2}-y^{2}}$, the $\mathrm{Cu}_{l} 3 d_{3 z^{2}-r^{2}}$ and $\mathrm{Cu}_{r} 3 d_{3 z^{2}-r^{2}}$ orbitals, and four virtuals. This is a 9 electrons/ 10 orbitals CAS, with about fifty thousand determinants.

For the minimum-energy state with stretched $\mathrm{Cu}_{l}-\mathrm{O}_{b}$ bonds and a $\mathrm{Cu}_{l} 3 d_{x^{2}-y^{2}}^{2}$ electron configuration, for example, those four correlating orbitals taken from the virtual orbital space in order to enlarge the minimal CAS have $\mathrm{Cu}_{l} 3 d_{x^{2}-y^{2}}^{\prime}, \mathrm{Cu}_{l} 4 s, \mathrm{Cu}_{r} 3 d_{3 z^{2}-r^{2}}^{\prime}$ and $\mathrm{Cu}_{r}$ $3 d_{x^{2}-y^{2}}^{\prime}$ character. There is some mixing between the $\mathrm{Cu}_{l}$ $3 d_{3 z^{2}-r^{2}}$ and $4 s$ basis functions. The occupation of the $\mathrm{Cu}_{l} 3 d_{3 z^{2}-r^{2}}$-like orbital is 1.97 and the predominantly $\mathrm{Cu}_{l} 4 s$ orbital has an occupation number of 0.03 . There are also large contributions of the $\mathrm{Cu} 4 s$ functions to some of the other active orbitals. The $\mathrm{Cu}_{r} 3 d_{3 z^{2}-r^{2}}$ and $3 d_{3 z^{2}-r^{2}}^{\prime}$ orbitals have occupation numbers of 1.99 and 0.01 , respectively. The occupation numbers of the $\mathrm{Cu}_{l}$ $3 d_{x^{2}-y^{2}}^{\prime}$ and $\mathrm{Cu}_{r} 3 d_{x^{2}-y^{2}}^{\prime}$ orbitals are somewhat smaller than 0.01.

Inclusion of the diffuse $d^{\prime}$ orbitals into the active space is necessary for describing changes in the radial charge distribution for different $d^{n}$ configurations in the multiconfigurational wave-function. For free TM atoms (ions), this is a correlation effect involving pairs of electrons in the $4 s^{2} 3 d^{n}, 4 s 3 d^{n+1}$, and $3 d^{n+2}$ states [28]. Occupation numbers as large as 0.1 for the $d^{\prime}$ orbitals of the $\mathrm{Ni}$ atom [31] demonstrate that a careful analysis is needed when dealing with TM atoms or TM compounds. In the copper oxide systems, the different $d^{n}$ configurations may be related to both on-site $\mathrm{Cu} 3 d-4 s$ and inter-site, $\mathrm{Cu}-\mathrm{Cu}$ and $\mathrm{O}-\mathrm{Cu}$, excitations. Radial, or "breathing", correlation effects may also occur for the O $2 p$ orbitals. When the ligand to metal charge transfer excitations are relevant 32], a highly accurate CAS SCF wave-function would require the inclusion of two extra sets of orbitals into the active space, ligand $2 p$ orbitals involved in the $\mathrm{O} 2 p-\mathrm{Cu} 3 d$ bonding plus the corresponding $2 p^{\prime}$ orbitals 25, 32]. Such an extension of the active space is not possible for our cluster, due to the high computational cost. Nevertheless, the low occupation numbers of the $3 d_{x^{2}-y^{2}}^{\prime}$ orbitals, see the preceding paragraph, suggest that the essential near-degeneracy correlation effects are already included in our model. It will be shown in the next section that the role of the $\mathrm{O} 2 p^{\prime}$ orbitals becomes important in the hole-doped case.

The potential energy landscape when stretching the $\mathrm{Cu}_{l}-\mathrm{O}_{b}$ or $\mathrm{Cu}_{r}-\mathrm{O}_{b}$ bonds is shown in Fig.3. The middle point on the horizontal axis corresponds to the undistorted cluster. From each minimum-energy configuration, the two $\mathrm{O}_{b}$ ions on the $\mathrm{Cu} 3 d_{x^{2}-y^{2}}^{2}$ plaquette are 


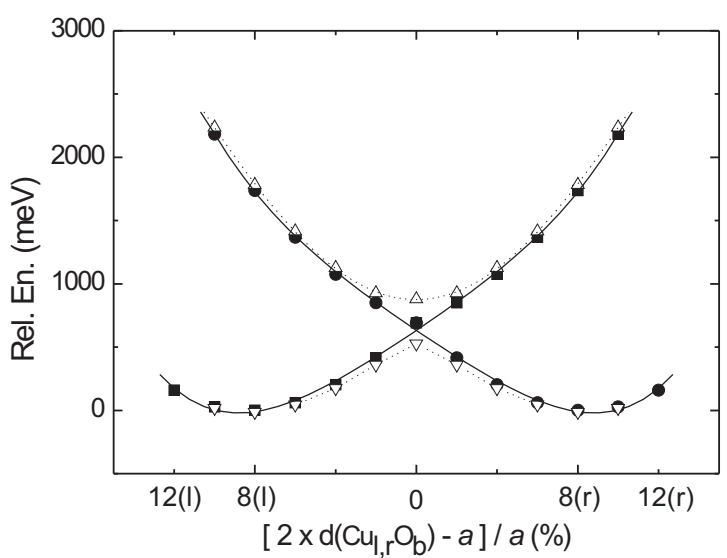

FIG. 3: Results of static, total energy calculations for an electron-doped, 4-plaquette cluster, see text and Fig.2. The filled symbols are the CASSCF data. The lowest points correspond to $\mathrm{Cu}_{l} 3 d_{x^{2}-y^{2}}^{2}$ or $\mathrm{Cu}_{r} 3 d_{x^{2}-y^{2}}^{2}$ configurations on plaquettes with $\mathrm{Cu}-\mathrm{O}_{b}$ distances larger by $8 \%$. For geometries where the $\mathrm{Cu}-\mathrm{O}_{b}$ bonds on the adjacent plaquette are stretched by more than $12 \%$, an 1-electron $\mathrm{Cu}_{l} d_{x^{2}-y^{2}}$ $\mathrm{Cu}_{r} d_{x^{2}-y^{2}}$ charge transfer occurs. The dotted curves are obtained by CAS SI calculations. The CAS SCF energies for the structures with $\mathrm{CuO}_{b}$ bonds stretched by $8 \%$ on one of the $\mathrm{Cu}_{l} \mathrm{O}_{4}$ or $\mathrm{Cu}_{r} \mathrm{O}_{4}$ plaquettes are taken as reference. $a$ is the in-plane lattice constant.

shifted back to the undistorted structure in steps of $2 \%$ of the high-symmetry $\mathrm{Cu}-\mathrm{O}$ distance. The orbitals from the previous step are used each time as starting orbitals. Once the undistorted geometry is reached we start stretching the $\mathrm{Cu}-\mathrm{O}_{b}$ bonds on the adjacent plaquette, see Fig.2. Each curve in Fig.3 is obtained by following this procedure. It turns out that the initial electronic wave-function remains largely unchanged for a relatively broad range of $\mathrm{Cu}-\mathrm{O}_{b}$ distortions. Its character changes abruptly when the $\mathrm{Cu}-\mathrm{O}_{b}$ bonds on the adjacent plaquette are elongated by about $12 \%$. Only for such a geometry the doped electron is transferred to the other $d_{x^{2}-y^{2}}$ orbital.

We list in Table I Mulliken populations of atomic orbitals on the $\mathrm{Cu}_{l} \mathrm{O}_{4}$ and $\mathrm{Cu}_{r} \mathrm{O}_{4}$ plaquettes for the $\mathrm{Cu}_{l}$ $d_{x^{2}-y^{2}}^{2}$ lowest energy configuration. The charges associated with the $\mathrm{O} 2 s$ and $\mathrm{O} 2 p_{x} / 2 p_{y}$ functions overlapping the $\mathrm{Cu} d_{x^{2}-y^{2}}$ orbitals are sensibly smaller than 2.00, even on the $d_{x^{2}-y^{2}}^{2}$ plaquette. The "missing" charge is in the $\mathrm{Cu} 4 s$ and $4 p$ orbitals. This deviation from the fully ionic picture was previously pointed out in Ref. [2], for example. Between the two plaquettes, the major differences are related to the populations of the $\mathrm{Cu} 3 d_{x^{2}-y^{2}}$, $3 d_{3 z^{2}-r^{2}}, 4 s, 4 p$, and $\mathrm{O}_{a} 2 p_{x}$ orbitals.

If the matrix elements between the $\mathrm{Cu}_{l} d_{x^{2}-y^{2}}^{2}$ and $\mathrm{Cu}_{r}$ $d_{x^{2}-y^{2}}^{2}$ electronic wave-functions are ignored, the adiabatic potential energy curves for the O-ion half-breathing displacements discussed above intersect at the point associated to the undistorted cluster, see Fig.3. However, if the electronic structures are allowed to relax by mixing of the $\mathrm{Cu}_{l} d_{x^{2}-y^{2}}^{2}$ and $\mathrm{Cu}_{r} d_{x^{2}-y^{2}}^{2}$ configurations, no intersection would occur. A so-called avoided crossing is expected for the potential curves. The matrix elements between non-orthogonal, separately optimized CAS SCF wave-functions can be obtained by State Interaction (SI) calculations 33. The potential energy curves corresponding to the CAS SI states are shown in Fig. 3 by dashed lines. The interaction between the nonorthogonal states is not too large, with energetic effects of not more than few tenths of $\mathrm{eV}$. The weights of the nonorthogonal states in the lowest CAS SI root are both $1 / 2$ at the middle point. For chain bonds stretched by $8 \%$ on one of the plaquettes, the weights are 0.99 and 0.01 . The energy barrier associated with the lower CAS SI curve is $540 \mathrm{meV}$.

For a more accurate estimate of the size of the energy barrier, polarization effects beyond the boundaries of the linear, 4-plaquette cluster should also be taken into account. Test calculations on a 5 -plaquette cluster where all four nearest neighbor plaquettes of a "central" $\mathrm{Cu}_{c}$ $d_{x^{2}-y^{2}}^{2}$ ion are treated at the all-electron level show indeed that such effects are important. We used for these calculations $D_{2 h}$ symmetry restrictions and the following basis sets [22]: $\mathrm{Cu} 21 s 15 p 10 d 6 f / 5 s 4 p 3 d 2 f$ for the five TM ions, O $14 s 9 p 4 d / 4 s 3 p 2 d$ for the ligands bridging two cations and O $14 s 9 p / 4 s 3 p$ for the other twelve oxygens. For a $\mathrm{Cu}_{c} d_{x^{2}-y^{2}}^{2}$ configuration on the plaquette at the center of the cluster, the CAS SCF energy difference between the undistorted structure and the geometry where the $\mathrm{Cu}_{c}-\mathrm{O}$ distances along the $y$ axis are longer by $8 \%$ is $130 \mathrm{meV}$ lower than for the linear, 4-plaquette cluster. Applying such a correction to the CAS SI results, the energy barrier would decrease to about $400 \mathrm{meV}$. Longer range polarization effects should further lower this value.

We also performed calculations on a 6 -plaquette linear cluster with two doped electrons. The following contractions were applied for the $\mathrm{Cu}$ and $\mathrm{O}$ basis sets: $\mathrm{Cu} 21 s 15 p 10 d / 5 s 4 p 3 d$ and $\mathrm{O} 14 s 9 p / 4 s 3 p$ [22]. Groundstate Mulliken populations for the geometry with $\mathrm{Cu}-\mathrm{O}_{b}$ bonds stretched by $8 \%$ on the $\mathrm{Cu}_{l l} \mathrm{O}_{4}$ and $\mathrm{Cu}_{r r} \mathrm{O}_{4}$ plaquettes are given in Table II. The results were obtained with an active space including 12 electrons and 12 orbitals. For this geometry and choice of the multiconfigurational space, the CAS SCF active orbitals were identified as six $\mathrm{Cu} 3 d_{x^{2}-y^{2}}$ orbitals, the $\mathrm{Cu}_{l l} 3 d_{3 z^{2}-r^{2}}$, $4 s$ and $\mathrm{Cu}_{r r} 3 d_{3 z^{2}-r^{2}}$, $4 s$ plus two correlating $\mathrm{Cu}_{l l} 3 d_{x^{2}-y^{2}}^{\prime}$ and $\mathrm{Cu}_{r r} 3 d_{x^{2}-y^{2}}^{\prime}$. The occupation numbers of these active orbitals are similar to those found for the smaller cluster. The Mulliken populations are very similar, too. The results show thus that localized $3 d_{x^{2}-y^{2}}^{2}$ states are also obtained in larger clusters and for more than a single doped electron. For the electron-doped case, an analysis of inter-carrier couplings by CAS SCF calculations on such larger clusters will be provided in a forthcoming publication. In the case of hole-doping, preliminary CAS SCF 
TABLE I: Mulliken charges per centre and basis function type for the minimum-energy $\mathrm{Cu}_{l} d_{x^{2}-y^{2}}^{2}$ configuration on an electrondoped, 4-plaquette linear cluster. The $\mathrm{Cu}_{l}-\mathrm{O}_{b}$ bonds are stretched by $8 \%$. Values for the $\mathrm{Cu}_{l} \mathrm{O}_{4}$ and $\mathrm{Cu}_{r} \mathrm{O}_{4}$ plaquettes are listed. The Mulliken populations on the other plaquettes, $\mathrm{Cu}_{l l} \mathrm{O}_{4}$ and $\mathrm{Cu}_{r r} \mathrm{O}_{4}$, are very similar to those on $\mathrm{Cu}_{r} \mathrm{O}_{4}$. The charge of each $3 d t_{2 g}$ atomic orbital is 2.0 . There is also some charge associated with the $\mathrm{Cu} f$ and $\mathrm{O} d$ functions, not shown in the table.

\begin{tabular}{lrrrrrr}
\hline \hline & $\mathrm{O}_{b}^{l}$ & $\mathrm{O}_{a}^{l}$ & $\mathrm{Cu}_{l}$ & $\mathrm{O}_{b}^{c}$ & $\mathrm{Cu}_{r}$ & $\mathrm{O}_{a}^{r}$ \\
\hline $\mathrm{O} 2 s, \mathrm{Cu} 4 s$ & 1.68 & 1.83 & 0.60 & 1.68 & 0.67 & 1.82 \\
$\mathrm{O} 2 p_{x}, \mathrm{Cu} 4 p_{x}$ & 1.99 & 1.96 & 0.14 & 1.99 & 0.22 & 1.89 \\
$\mathrm{O} 2 p_{y}, \mathrm{Cu} 4 p_{y}$ & 1.75 & 2.00 & 0.09 & 1.74 & 0.23 & 2.00 \\
$\mathrm{O} 2 p_{z}, \mathrm{Cu} 4 p_{z}$ & 1.87 & 1.97 & 0.08 & 1.87 & 0.17 & 1.99 \\
$\mathrm{Cu} d_{3 z^{2}-r^{2}}$ & & & 1.83 & & 1.96 & 1.70 \\
$\mathrm{Cu} d_{x^{2}-y^{2}}$ & & & & & 1.18 \\
Total el. charge & 9.30 & 9.77 & 28.00 & & 28.54 & 9.67 \\
\hline \hline
\end{tabular}

TABLE II: Mulliken charges per centre and basis function type for a 6-plaquette linear cluster with two doped electrons. The $\mathrm{Cu}_{l l}-\mathrm{O}_{b}$ and $\mathrm{Cu}_{r r}-\mathrm{O}_{b}$ bonds are stretched by $8 \%$. Values for $\mathrm{Cu}$ and $\mathrm{O}_{b}$ ions on the four central plaquettes, $\mathrm{Cu}_{l l} \mathrm{O}_{4}, \mathrm{Cu}_{l} \mathrm{O}_{4}$, $\mathrm{Cu}_{r} \mathrm{O}_{4}$, and $\mathrm{Cu}_{r r} \mathrm{O}_{4}$, are listed. The same notations as in Fig.2. are used.

\begin{tabular}{lccccccccc}
\hline \hline & $\mathrm{O}_{b}^{l l}$ & $\mathrm{Cu}_{l l}$ & $\mathrm{O}_{b}^{l}$ & $\mathrm{Cu}_{l}$ & $\mathrm{O}_{b}^{c}$ & $\mathrm{Cu}_{r}$ & $\mathrm{O}_{b}^{r}$ & $\mathrm{Cu}_{l l}$ & $\mathrm{O}_{b}^{r r}$ \\
\hline $\mathrm{O} 2 s, \mathrm{Cu} 4 s$ & 1.68 & 0.58 & 1.67 & 0.67 & 1.69 & 0.67 & 1.67 & 0.58 & 1.68 \\
$\mathrm{O} 2 p_{x}, \mathrm{Cu} 4 p_{x}$ & 1.99 & 0.12 & 1.99 & 0.22 & 1.99 & 0.22 & 1.99 & 0.12 & 1.99 \\
$\mathrm{O} 2 p_{y}, \mathrm{Cu} 4 p_{y}$ & 1.73 & 0.09 & 1.74 & 0.24 & 1.72 & 0.24 & 1.74 & 0.09 & 1.73 \\
$\mathrm{O} 2 p_{z}, \mathrm{Cu} 4 p_{z}$ & 1.86 & 0.08 & 1.87 & 0.17 & 1.89 & 0.17 & 1.87 & 0.08 & 1.86 \\
$\mathrm{Cu} d_{3 z^{2}-r^{2}}$ & & 1.82 & & 1.97 & & 1.97 & 1.82 & $\mathbf{2 . 0 0}$ \\
$\mathrm{Cu} d_{x^{2}-y^{2}}$ & & $\mathbf{2 . 0 0}$ & & 1.18 & & 1.18 & 9.27 & 28.73 \\
Total el. charge & 9.26 & 28.73 & 9.27 & 28.55 & 9.30 & 28.55 & 9.26 \\
\hline \hline
\end{tabular}

calculations on 2-oxygen-hole, 6-octahedra clusters reveal that the hole-hole interaction is large. Due to the strong mutual repulsion within the O $2 p$ "band", the two holes are pushed to the boundaries of the cluster and meaningful CAS SCF results cannot be obtained in this situation. However, inter-carrier correlations for the hole-doped case are addressed in the next section by a model Hamiltonian approach that enables calculations on chains of few tens of plaquettes.

The existence of a local double-well potential like that shown in Fig.3 for $\mathrm{Cu}-\mathrm{O}$ bond-length distortions in both n-doped and p-doped layered $\mathrm{Cu}$ oxides was predicted by Goodenough 12]. Goodenough also suggested that around optimal doping the doped carriers display some particular (dynamic) ordering within each layer, perhaps an arrangement with alternating undoped and 1/3-doped chains of plaquettes. The coupling between these ordered electrons/holes and in-plane O-ion halfbreathing phonons is believed to be at the heart of hightemperature superconductivity 11, 12, 13. Evidence for stripe formation in electron-doped cuprates was recently reported in Ref. 34], for example.

\section{HOLE-DOPED SYSTEM}

Multiconfiguration calculations for hole-doped clusters have been performed in Ref. 6]. However, a minimal active space was applied in that study, with a single orbital for each $\mathrm{Cu} 3 d$ or $\mathrm{O} 2 p$ hole. In this section we report results of CASSCF calculations with larger active spaces. We use the same 4-octahedra linear cluster and the same (static) embedding as in Ref. 6], with the lattice parameters of $\mathrm{La}_{1.85} \mathrm{Sr}_{1.85} \mathrm{CuO}_{4}$ [35]. The basis sets are also the same, $\mathrm{Cu} 21 s 15 p 10 d / 5 s 4 p 3 d$, O $14 s 9 p 4 d / 4 s 3 p 1 d$ for the bridging $\mathrm{O}_{b}$ oxygens, and $\mathrm{O} 14 s 9 p / 4 s 3 p$ for the other oxygens [22]. As already mentioned in Introduction, the CASSCF results indicate that ZR type singlet states are only formed on $\mathrm{CuO}_{4}$ plaquettes with $\mathrm{Cu}-\mathrm{O}$ bonds shorter than the average $\mathrm{Cu}-\mathrm{O}$ distance deduced from experiment. On the other hand, for the undistorted geometry the doped hole has the largest weight onto a single ligand and in the simplest picture the dominant contribution to the wave-function comes from a $\ldots-\mathrm{Cu} d^{9}$ $\mathrm{O} p^{5}-\mathrm{Cu} d^{9}-\ldots$ configuration [36]. The latter state is few hundreds meV higher in energy than the former. Also for the hole-doped case, O-ion half-breathing displacements determine thus a multi-well energy landscape and can induce charge transfer along a chain of $\mathrm{CuO}_{4}$ plaquettes. We note that geometry optimizations for transition metal oxides like $\mathrm{MnO}$ and $\mathrm{NiO}$ at the Hartree-Fock (HF) level [37, 38] slightly overestimate the interatomic distances. This means that the multi-well energy landscape would also be obtained with such HF optimized lattice parameters 39]. For the electron-doped case, the usual overestimation of the lattice constants, $2-4 \%$ [37, 38], is significantly lower than the range of the $\mathrm{Cu}-\mathrm{O}$ bond distortions associated with the multi-well energy curve, 
TABLE III: Major contributions of the oxygen $2 p$ and metal $3 d$ atomic orbitals to those active (natural) orbitals implying $2 p-2 p$ and $2 p-3 d$ bonding on the $\mathrm{Cu}_{l} \mathrm{O}_{4}$ and $\mathrm{Cu}_{r} \mathrm{O}_{4}$ plaquettes. CAS SCF results for a hole-doped, 4-plaquette linear cluster. Coefficients smaller than 0.20 are not listed. For each geometry, the nature of the other active orbitals is discussed in the text.

\begin{tabular}{|c|c|c|c|c|c|c|c|c|}
\hline \multirow{2}{*}{$\begin{array}{l}\text { State: } \\
\text { Orb. Occ. No.: }\end{array}$} & \multicolumn{4}{|c|}{$\mathrm{O}_{b}^{c}$-hole, undistorted cluster } & \multicolumn{4}{|c|}{$\mathrm{Cu}_{l} \mathrm{O}_{4} \mathrm{ZR}$, distorted $\mathrm{Cu}_{l}-\mathrm{O}_{b}$ bonds ${ }^{a}$} \\
\hline & 1.99 & 1.96 & 1.84 & 0.21 & 1.99 & 1.97 & 1.90 & 0.13 \\
\hline $\mathrm{O}_{b}^{l} p_{y}$ & & 0.54 & 0.22 & 0.37 & 0.29 & 0.67 & 0.41 & 0.42 \\
\hline $\mathrm{O}_{a}^{l} p_{x}(\mathrm{x} 2)$ & \pm 0.20 & \pm 0.25 & \pm 0.26 & \pm 0.31 & $\mp 0.42$ & & \pm 0.29 & \pm 0.29 \\
\hline $\mathrm{Cu}_{l} d_{x^{2}-y^{2}}$ & & & -0.61 & 0.74 & & & -0.63 & 0.77 \\
\hline $\mathrm{O}_{b}^{c} p_{y}$ & 0.37 & 0.51 & -0.56 & -0.47 & -0.29 & 0.67 & -0.41 & -0.42 \\
\hline $\mathrm{Cu}_{r} d_{x^{2}-y^{2}}$ & & & & -0.21 & & & & \\
\hline $\mathrm{O}_{a}^{r} p_{x}(\mathrm{x} 2)$ & $\mp 0.37$ & \pm 0.21 & & & & & & \\
\hline $\mathrm{O}_{b}^{r} p_{y}$ & 0.39 & & & & & & & \\
\hline
\end{tabular}

${ }^{a}$ The chain $\mathrm{Cu}_{l}-\mathrm{O}_{b}$ bonds are $6 \%$ shorter.

$\pm 8 \%$.

As in the previous section, we added to the minimal active space orbitals from both the inactive, doubly occupied set and the virtual space. Trial calculations showed that the most important near-degeneracy correlation effects are already accounted for with extensions of only few orbitals. In Tables III, IV, and V we show results obtained from CAS SCF calculations with 13 electrons and 12 orbitals in the active space. Compared to the minimal (i.e., 5 electrons $/ 5$ orbitals) CAS, four inactive and three virtual orbitals were moved into the active set. Depending on the input starting orbitals, different CAS SCF solutions can be obtained for the undistorted cluster, with the doped hole having the largest weight onto one of the three bridging oxygens, $\mathrm{O}_{b}^{l}, \mathrm{O}_{b}^{c}$ or $\mathrm{O}_{b}^{r}$. The largest contributions to those active orbitals implying $2 p-2 p$ and $3 d-2 p$ bonding on the central $\mathrm{Cu}_{l} \mathrm{O}_{4}$ and $\mathrm{Cu}_{r} \mathrm{O}_{4}$ plaquettes for the CASSCF solution with the doped hole having the largest weight onto the $\mathrm{O}_{b}^{c}$ ligand are illustrated in Table III. We also display in Table III the occupation numbers of those orbitals. There are other three active orbitals with $\mathrm{Cu}_{l l}, \mathrm{Cu}_{r}$, and $\mathrm{Cu}_{r r} 3 d_{x^{2}-y^{2}}$ character and occupation numbers of nearly 1. For this CAS SCF solution, the remaining active orbitals have $\mathrm{O}_{b}^{c}$ $2 s, \mathrm{Cu}_{l} 3 d_{3 z^{2}-r^{2}}, \mathrm{O}_{b}^{c} 2 p_{y}^{\prime}, \mathrm{Cu}_{l} 4 s-\mathrm{O}_{b}^{c} 2 s^{\prime}-\mathrm{Cu}_{r} 4 s$, and $\mathrm{Cu}_{l} 3 d_{3 z^{2}-r^{2}}^{\prime}$ character. Their occupation numbers are $1.999,1.98,0.02,0.01$, and 0.01 , respectively.

The Mulliken electron populations for the relevant atomic functions on the $\mathrm{Cu}_{l} \mathrm{O}_{4}$ and $\mathrm{Cu}_{r} \mathrm{O}_{4}$ plaquettes are listed in Table IV. Compared to the minimal-CAS calculations from Ref. [6] , the charge disproportion between the $\left(\mathrm{O}_{b}^{l} 2 p_{y}, \mathrm{O}_{a}^{l} 2 p_{x}\right)$ and $\mathrm{O}_{b}^{c} 2 p_{y}$ orbitals is significantly smaller. The difference between the $\mathrm{O}_{b}^{l} 2 p_{y}$ and $\mathrm{O}_{b}^{c} 2 p_{y}$ Mulliken charges, for example, is 0.13 instead of 0.50 . We also note the $\mathrm{Cu}_{l}-\mathrm{O}_{b}^{c}-\mathrm{Cu}_{r}$ asymmetry. A symmetry equivalent solution, with a pair of bonding and antibond-

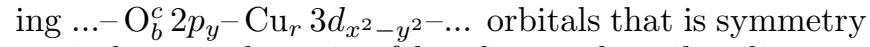
equivalent to the pair of bonding and antibonding ...$\mathrm{Cu}_{l} 3 d_{x^{2}-y^{2}}-\mathrm{O}_{b}^{c} 2 p_{y}-\ldots$ orbitals in Table III (the third and fourth columns) can also be obtained. The CAS SCF wave-function changes its character from one type to the other for displacements of the $\mathrm{O}_{b}^{c}$ ion of $\pm 0.8 \%$ around the middle position. This is very different in comparison with the electron-doped case in the previous section, where the character of the CAS SCF wave-function remains nearly unchanged, i.e. either $\mathrm{Cu}_{l} d_{x^{2}-y^{2}}^{2}$ or $\mathrm{Cu}_{r} d_{x^{2}-y^{2}}^{2}$, for distortions of up to $\pm 12 \%$ of the $\mathrm{Cu}-\mathrm{O}_{b}$ bonds.

With the larger active space, the ZR like state with shorter $\mathrm{Cu}-\mathrm{O}_{b}$ distances (6\% shorter) on one of the $\mathrm{Cu}_{l} \mathrm{O}_{4}$ or $\mathrm{Cu}_{r} \mathrm{O}_{4}$ plaquettes is lower in energy by about $460 \mathrm{meV}$. Major contributions to the relevant $2 p-3 d$ active orbitals are shown in Table III. The doped hole is now approximately equally distributed over the $\mathrm{O}_{b} p_{y}$ and $\mathrm{O}_{a}$ $p_{x}$ atomic orbitals on the distorted plaquette, see the pair of $d-p$ bonding and antibonding orbitals with lower occupation numbers in Table III. As above, there are three active orbitals with $\mathrm{Cu}_{l l}, \mathrm{Cu}_{r}$, and $\mathrm{Cu}_{r r} 3 d_{x^{2}-y^{2}}$ character and occupation numbers of roughly 1 . The other active orbitals have $\mathrm{Cu}_{l} 3 p_{y}, \mathrm{Cu}_{l} 3 d_{3 z^{2}-r^{2}}, \mathrm{Cu}_{l} 4 s-\mathrm{O}_{b / a} 2 s^{\prime} / 2 p^{\prime}$, $\mathrm{O}_{b}^{l} 2 p_{y}^{\prime}-\mathrm{Cu}_{l} 4 p_{y}-\mathrm{O}_{b}^{c} 2 p_{y}^{\prime}$, and $\mathrm{Cu}_{l} 3 d_{3 z^{2}-r^{2}}^{\prime}$ character and occupation numbers of $1.999,1.98,0.01,0.01$, and 0.01 , respectively. It can be seen that the nature of some of these orbitals changes from the undistorted to the distorted cluster. The CASSCF results indicate that in the latter case the $\mathrm{O} 2 s / 2 p-\mathrm{Cu} 4 s / 4 p-\mathrm{O} 2 s / 2 p$ interactions on the same plaquette are more important than the inter-plaquette $\mathrm{Cu} 4 s / 4 p-\mathrm{O} 2 s / 2 p-\mathrm{Cu} 4 s / 4 p$ interactions for the former. Mulliken charges associated with such a ZR like state are listed in Table V. The comparison between the results displayed in Table V (or Table IV) and those obtained for the electron-doped $\mathrm{Cu}_{l} \mathrm{O}_{4}$ plaquette in Table I reveals a remarkable feature: due to strong charge redistribution effects within the $\mathrm{Cu}_{l} 3 d_{3 z^{2}-r^{2}}, 4 s$, $4 p$ and $\mathrm{O} 2 s, 2 p$ orbitals, the total electronic charge associated with the $\mathrm{Cu}_{l} 3 d_{x^{2}-y^{2}}^{1}$ ion in Table V (or Table IV) is nearly as high as that associated with the $\mathrm{Cu}_{l} 3 d_{x^{2}-y^{2}}^{2}$ configuration from Table I, 28.7 versus 28.8. It is well known that the Mulliken charges may be misleading in cases where strong inter-atomic orbital overlap occurs. Still, a variation of nearly one elementary charge within the $\mathrm{Cu} 4 s, 4 p$ shell cannot be an artifact.

The adiabatic energy landscape for $\mathrm{Cu}-\mathrm{O}_{b}$ bond-length distortions on the two central plaquettes is shown in Fig.4. The points on the full lines are the minimal-CAS 
TABLE IV: Mulliken charges per centre and basis function type for a broken-symmetry oxygen-hole state on a 4-octahedra linear cluster. The doped hole has the largest weight on the $\mathrm{O}_{b}^{c}$ ion. Charges for the central $\mathrm{Cu}_{l} \mathrm{O}_{4}$ and $\mathrm{Cu}_{r} \mathrm{O}_{4}$ plaquettes are listed. The $\mathrm{Cu}-\mathrm{O}$ distances are all the same.

\begin{tabular}{lcccccc}
\hline \hline & $\mathrm{O}_{b}^{l}$ & $\mathrm{O}_{a}^{l}$ & $\mathrm{Cu}_{l}$ & $\mathrm{O}_{b}^{c}$ & $\mathrm{Cu}_{r}$ & $\mathrm{O}_{a}^{r}$ \\
\hline $\mathrm{O} 2 s, \mathrm{Cu} 4 s$ & 1.59 & 1.81 & 0.77 & 1.58 & 0.67 & 1.81 \\
$\mathrm{O} 2 p_{x}, \mathrm{Cu} 4 p_{x}$ & 2.00 & $\mathbf{1 . 6 5}$ & 0.28 & 2.00 & 0.26 & 1.81 \\
$\mathrm{O} 2 p_{y}, \mathrm{Cu} 4 p_{y}$ & $\mathbf{1 . 6 5}$ & 2.00 & 0.22 & $\mathbf{1 . 5 1}$ & 0.17 & 2.00 \\
$\mathrm{O} 2 p_{z}, \mathrm{Cu} 4 p_{z}$ & 1.93 & 1.97 & 0.25 & 1.92 & 0.23 & 1.97 \\
$\mathrm{Cu} d_{3 z^{2}-r^{2}}$ & & & 2.00 & & 2.00 & 1.83 \\
$\mathrm{Cu} d_{x^{2}-y^{2}}$ & & & 1.06 & & 1.15 & \\
Total el. charge & 9.19 & 9.42 & 28.70 & 9.03 & 28.57 & 9.59 \\
\hline \hline
\end{tabular}

TABLE V: Mulliken charges per centre and basis function for a minimum-energy ZR type configuration on the $\mathrm{Cu}_{l} \mathrm{O}_{4} \mathrm{plaquette}$ CAS SCF results for an 1-doped-hole, 4-octahedra linear cluster, see text. The $\mathrm{Cu}_{l}-\mathrm{O}_{b}$ bonds are shorter by $6 \%$. Values for the central $\mathrm{Cu}_{l} \mathrm{O}_{4}$ and $\mathrm{Cu}_{r} \mathrm{O}_{4}$ plaquettes are listed.

\begin{tabular}{lcccccc}
\hline \hline & $\mathrm{O}_{b}^{l}$ & $\mathrm{O}_{a}^{l}$ & $\mathrm{Cu}_{l}$ & $\mathrm{O}_{b}^{c}$ & $\mathrm{Cu}_{r}$ & $\mathrm{O}_{a}^{r}$ \\
\hline $\mathrm{O} 2 s, \mathrm{Cu} 4 s$ & 1.57 & 1.81 & 0.82 & 1.57 & 0.64 & 1.80 \\
$\mathrm{O} 2 p_{x}, \mathrm{Cu} 4 p_{x}$ & 2.00 & $\mathbf{1 . 6 5}$ & 0.29 & 2.00 & 0.25 & 1.83 \\
$\mathrm{O} 2 p_{y}, \mathrm{Cu} 4 p_{y}$ & $\mathbf{1 . 5 9}$ & 2.00 & 0.25 & $\mathbf{1 . 5 9}$ & 0.15 & 2.00 \\
$\mathrm{O} 2 p_{z}, \mathrm{Cu} 4 p_{z}$ & 1.92 & 1.97 & 0.25 & 1.92 & 0.23 & 1.97 \\
$\mathrm{Cu} d_{3 z^{2}-r^{2}}$ & & & 2.00 & & 2.00 & 1.84 \\
$\mathrm{Cu} d_{x^{2}-y^{2}}$ & & & 1.02 & & 1.16 & \\
Total el. charge & 9.10 & 9.43 & 28.74 & 9.09 & 28.53 & 9.61 \\
\hline \hline
\end{tabular}

results already published in Ref. 6]. The energy maxima correspond to states with dominant $\mathrm{O}_{b}$-hole character in the undistorted structure. The two minima are related to $\mathrm{ZR}$ like states where the $\mathrm{Cu}-\mathrm{O}_{b}$ distances on the $\mathrm{Cu}_{l} \mathrm{O}_{4}$ plaquette (the left hand minimum) or the $\mathrm{Cu}-$ $\mathrm{O}_{b}$ distances on the $\mathrm{Cu}_{r} \mathrm{O}_{4}$ plaquette (the right hand minimum) are $6 \%$ shorter. From each minimum-energy structural configuration, the two $\mathrm{O}_{b}$ ligands are shifted back to the undistorted geometry in steps of $1 \%$ of the high-symmetry $\mathrm{Cu}-\mathrm{O}$ bond length. These states are shown with black dots. The last point on each of the two curves corresponds to a configuration where the $\mathrm{Cu}^{-}$ $\mathrm{O}_{b}$ distances on either the $\mathrm{Cu}_{l l} \mathrm{O}_{4}$ or $\mathrm{Cu}_{r r} \mathrm{O}_{4}$ plaquette are shorter, by $1 \%$. Other calculations were carried out for the case where the $\mathrm{Cu}-\mathrm{O}_{b}$ bonds on the $\mathrm{Cu}_{l} \mathrm{O}_{4}$ and $\mathrm{Cu}_{r} \mathrm{O}_{4}$ plaquettes are gradually shortened starting from the undistorted structure and using the orbitals of the $\mathrm{O}_{b}^{c}$-hole state as starting orbitals. Due to convergence problems, only few points could be obtained. Those points are shown as black triangles.

Relative energies from CAS SCF calculations with 13 electrons and 12 orbitals in the active space are shown in Fig. 4 only for the ZR like states with $\mathrm{Cu}-\mathrm{O}_{b}$ distances shorter by $6 \%$ and the states with undistorted bonds. These points are marked with filled squares. The energy lowering induced by the use of a larger active space is of the order of $1.5-2.0 \mathrm{eV}$. The effect is stronger for the distorted configurations. For convenience, the larger-CAS energies are shifted upwards in Fig. 4 by $1.6 \mathrm{eV}$. We note that the active orbitals and the CI vectors for the states with dominant $\mathrm{O}_{b}^{l}$ or $\mathrm{O}_{b}^{r}$-hole character are similar to those of the state where the hole has the largest weight on the $\mathrm{O}_{b}^{c}$ ligand, with a "shift" of one plaquette to the left or to the right, respectively.

Separately optimized, broken-symmetry wavefunctions like the different O-hole solutions for the undistorted geometry are non-orthogonal and interacting. Orthogonal, non-interacting eigenstates can be obtained by SI calculations. However, the situation is now somewhat different as compared to the SI calculations for the electron-doped cluster. First, the way the energy landscape was drawn is different. The two sides of a well in the hole-doped case correspond to identical positions of the $\mathrm{O}_{b}$ ions, although CASSCF roots on different sides have different character, at least for small distortions. In the second place, the O-hole is "smoothly" transferred from one plaquette to the other, for distortions of about $1 \%$ of the $\mathrm{Cu}-\mathrm{O}$ bond(s). Matrix elements exist thus only between different O-hole states in the undistorted geometry or between states situated on different sides of the same well. We note that in the electron-doped cluster, matrix elements exist only between CASSCF states associated with different wells. Those matrix elements determine in the electron-doped case a so-called avoided crossing for the energy curves. The missing points for each of the two wells in Fig.4 could probably be obtained from calculations on clusters including several 1-hole, 3-octahedra units. For certain phases of the O-ion half-breathing vibrations on the neighboring plaquettes, see Fig.1, the O-hole on a given 3-octahedra unit should move "smoothly" from one side to the other. In fact, 
it is the longer range Coulomb and spin interactions that can induce indeed broken-symmetry oxygen-hole states in the infinite system. The collective, coherent "tunneling" of these quasi-localized oxygen holes along a multi-well energy landscape like that depicted in Fig.4 can lead to a traveling charge-density/spin-density wave as in the model proposed by Goodenough 12 .

The tendency towards the formation of quasi-localized oxygen-hole states on distorted $\mathrm{CuO}_{4}$ plaquettes is confirmed by model Hamiltonian calculations. We consider in the following paragraphs isolated chains of $\mathrm{CuO}_{4}$ plaquettes at $1 / 3$ hole-doping and construct a $p-d$, Hubbard type model:

$$
\begin{aligned}
H & =\epsilon_{d} \sum_{i \sigma} n_{i \sigma}+\epsilon_{p} \sum_{j \sigma} n_{j \sigma}+\sum_{<i j>\sigma} t_{p d}\left(d_{i \sigma}^{\dagger} p_{j \sigma}+\text { h.c. }\right) \\
& +\sum_{<j j^{\prime}>\sigma} t_{p p}\left(p_{j \sigma}^{\dagger} p_{j^{\prime} \sigma}+\text { h.c. }\right) \\
& +U_{d d} \sum_{i} n_{i \uparrow} n_{i \downarrow}+U_{p p} \sum_{j} n_{j \uparrow} n_{j \downarrow} \\
& +V_{p d} \sum_{<i j>\sigma \sigma^{\prime}} n_{i \sigma} n_{j \sigma^{\prime}}+V_{p p} \sum_{<j j^{\prime}>\sigma \sigma^{\prime}} n_{j \sigma} n_{j^{\prime} \sigma^{\prime}} .
\end{aligned}
$$

We neglect the apical ligands and restrict to a single orbital at each site: $\mathrm{Cu} 3 d_{x^{2}-y^{2}}$, bridging oxygen $\mathrm{O}_{b}$ $2 p_{y}$ orbitals, and oxygen $2 p_{x}$ orbitals at the other lig-

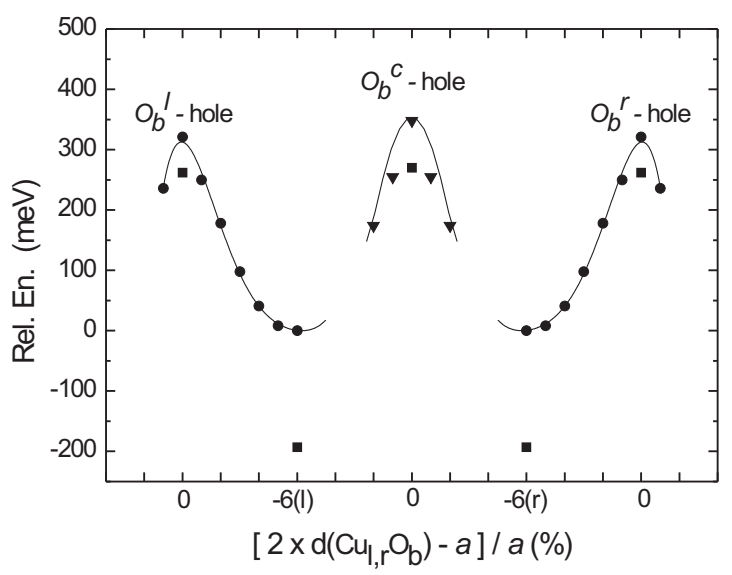

FIG. 4: Results of static, total energy calculations for a hole-doped, 4-plaquette cluster, see text. Filled circles and triangles are the minimal-CAS (i.e. 5 electrons/ 5 orbitals) results. The lowest points are related to ZR like states where the $\mathrm{Cu}_{l}-\mathrm{O}_{b}$ distances (the left hand minimum) or the $\mathrm{Cu}_{r}-$ $\mathrm{O}_{b}$ distances (the right hand minimum) are $6 \%$ shorter. The energy maxima correspond to broken-symmetry states in the undistorted geometry. The filled squares are CASSCF results with 13 electrons and 12 orbitals in the active space. The minimal-CAS energy of the ZR state is taken as reference. For convenience, the larger-CAS energies are shifted upwards by $1.6 \mathrm{eV}$. and sites, $\mathrm{O}_{a}$. We take into account the overlap integrals and Coulomb interactions between the nearest neighbor $d_{x^{2}-y^{2}}$ and $p_{y} / p_{x} \sigma$-orbitals and also between the second nearest neighbors $\mathrm{O}_{b} p_{y}$ and $\mathrm{O}_{a} p_{x}$. In the electron representation, we choose $\Delta=\epsilon_{d}-\epsilon_{p}=3.0$ [29] and set $t_{p d}^{x}=-1.2, t_{p d}^{y}=-1.2$, and $t_{p p}=0.5(\mathrm{eV})$. The values of the Coulomb repulsion parameters are chosen according to results of constrained LDA [40] and/or ab initio, explicitly correlated 2, 3, 4, 24 calculations: $U_{d d}=9.0$, $U_{p p}=5.0, V_{p d}=1.5$, and $V_{p p}=1.2(\mathrm{eV})$. Under the assumption of charge segregation into undoped and $1 / 3$ doped stripes [12], we also include for the $\mathrm{O}_{a}$ ligands inter-site Coulomb interactions due to electrons at $\mathrm{Cu}$ and $\mathrm{O}$ sites on the neighboring, undoped $\mathrm{Cu} d^{9}-\mathrm{O} p^{6}-\ldots$ chains. That is, the energy of the $\mathrm{O}_{a} p_{x}$ orbitals is shifted from $\epsilon_{p}=\epsilon_{p}^{y}=\epsilon_{d}-3$ to $\epsilon_{p}^{x}=\epsilon_{p}+V_{p d}+4 V_{p p}$.

Ground-state expectation values are obtained by density-matrix renormalization-group (DMRG) techniques [41]. For these calculations chains of $33 \mathrm{CuO}_{4}$ plaquettes, open-end boundary conditions, and $m=1200$ states to build the DMRG basis were used. The typical discarded weight is of the order $10^{-11}-10^{-10}$ or lower.

Occupation numbers of $p$ and $d$ orbitals on the 3plaquette unit at the middle of the 33-plaquette chain are listed in Table VI (first column). The $\mathrm{Cu}$ site right at the middle is labeled $\mathrm{Cu}_{c}$. Along the $\mathrm{Cu}-\mathrm{O}_{b}-\ldots$ chain, the next sites to the left and to the right are denoted $\mathrm{O}_{b}^{l}, \mathrm{Cu}_{l}, \mathrm{O}_{b}^{l l}$ and $\mathrm{O}_{b}^{r}, \mathrm{Cu}_{r}, \mathrm{O}_{b}^{r r}$, respectively. For each of the $\mathrm{Cu}$ ions mentioned above there is also a pair of $\mathrm{O}_{a}$ sites. These ligands are labeled $\mathrm{O}_{a}^{l}, \mathrm{O}_{a}^{c}$ or $\mathrm{O}_{a}^{r}$. The occupation numbers of the $d$ orbitals are comparable to those obtained in the CAS SCF calculations for the small cluster. However, since the $\mathrm{O} 2 s, 2 p \rightarrow \mathrm{Cu} 4 s, 4 p$ (back) charge transfer mentioned above is not taken into account in our $p-d$ model Hamiltonian, the electron occupation of the $p$ orbitals is somewhat larger. Also, compared to the $a b$ initio results, there is no charge disproportion along the undistorted chain of $\mathrm{CuO}_{4}$ plaquettes. This may be attributed to various effects. The most important should be the neglect of longer range Coulomb interactions in the $p-d$ model Hamiltonian and the orbital optimization for the CASSCF wave-function. The fact that the variational optimization of the "molecular" orbitals can lead to broken-symmetry solutions was shown for a simple model Hamiltonian by Martin [4].

Next, we simulate the shortening of the $\mathrm{Cu}-\mathrm{O}_{b}$ bonds on every third plaquette along the chain by introducing a modulation of the hopping matrix elements and inter-site Coulomb repulsion parameters: $t_{p d}^{\prime}(r)=t_{p d}(0)\left(r_{0} / r\right)^{7 / 2}$, $t_{p p}^{\prime}(r)=t_{p p}(0)\left(r_{0} / r\right)^{3}$ [42], and $V^{\prime}(r)=V(0)\left(r_{0} / r\right)$. Orbital occupation numbers are given in Table VI, for bonds shortened by $6 \%$ on the central plaquette on every 3 plaquette unit (second column) and for bonds shortened by $6 \%$ on the right-hand plaquette on every 3 -plaquette unit (third column). We also list the energies calculated for the three configurations, with the energy of the undistorted chain taken as reference. The results show that the energy of the system is lowered for the distorted config- 
TABLE VI: Orbital occupation numbers on the 3-plaquette unit at the middle of a chain of $33 \mathrm{CuO}_{4}$ plaquettes at $1 / 3$ hole doping. DMRG results for a $p-d$ extended Hubbard model, see text. Three different geometries are considered. In the distorted configurations, the $\mathrm{Cu}-\mathrm{O}_{b}$ bonds are shorter by $6 \%$ on every third plaquette. Each occupation number does not change by more than 0.02 for the next four "units". Relative energies are also given, for a whole chain.

\begin{tabular}{lccc}
\hline \hline & Undist. & Dist. $\mathrm{Cu}_{c}-\mathrm{O}_{b}$ & Dist. $\mathrm{Cu}_{r}-\mathrm{O}_{b}$ \\
\hline $\mathrm{O}_{b}^{l l} p_{y}$ & 1.75 & 1.80 & 1.71 \\
$\mathrm{Cu}_{l} d_{x^{2}-y^{2}}$ & 1.14 & 1.18 & 1.18 \\
$\mathrm{O}_{a}^{l} p_{x}$ & 1.89 & 1.93 & 1.93 \\
$\mathrm{O}_{b}^{l} p_{y}$ & 1.74 & $\mathbf{1 . 7 1}$ & 1.81 \\
$\mathrm{Cu}_{c} d_{x^{2}-y^{2}}$ & 1.13 & $\mathbf{1 . 0 4}$ & 1.18 \\
$\mathrm{O}_{a}^{c} p_{x}$ & 1.88 & $\mathbf{1 . 8 3}$ & 1.93 \\
$\mathrm{O}_{b}^{r} p_{y}$ & 1.74 & $\mathbf{1 . 7 1}$ & $\mathbf{1 . 7 1}$ \\
$\mathrm{Cu}_{r} d_{x^{2}-y^{2}}$ & 1.14 & 1.18 & $\mathbf{1 . 0 4}$ \\
$\mathrm{O}_{a}^{r} p_{x}$ & 1.89 & 1.93 & $\mathbf{1 . 8 3}$ \\
$\mathrm{O}_{b}^{r r} p_{y}$ & 1.75 & 1.80 & $\mathbf{1 . 7 1}$ \\
$\mathrm{Rel}$ En. $(\mathrm{eV})$ & 0 & -2.80 & -2.44 \\
\hline \hline
\end{tabular}

urations. The small energy difference between the two differently distorted structures, 2.80 versus $2.44 \mathrm{eV}$, is due to finite size effects. In addition, the charge redistribution within the 3 -plaquette unit(s) confirms the trend observed for the CAS SCF results on the smaller cluster: the oxygen holes tend to localize on the distorted plaquettes. Electron-phonon interactions and a softening of the half-breathing O-ion vibrations were predicted in the hole-doped regime by other authors as well [3], mostly on the basis of $t-J$ model Hamiltonian studies. With the present DMRG calculations for a $p-d$ extended Hubbard model, we found that the energetic effects associated with $\mathrm{Cu}-\mathrm{O}_{b}$ bond-length distortions on every third plaquette along a chain of several plaquettes at $1 / 3$-doping is of the order of hundred meV per each 3-plaquette "unit" (approximately $255 \mathrm{meV}$ for distortions of $6 \%$ ). These estimates are in line with the CAS SCF results discussed above. Nevertheless, the $a b$ initio calculations indicate a much stronger effect.

\section{CONCLUSIONS}

Results of $a b$ initio multiconfiguration calculations on clusters including few $\mathrm{CuO}_{4}$ plaquettes and also results of DMRG calculations for a $p-d$ extended Hubbard model with chains of few tens of plaquettes indicate the presence of strong anharmonic effects for the O-ion half-breathing deformations. In addition, for certain phases of the Oion vibrations, charge can be displaced along the $\mathrm{CuO}$ chain. These effects are observed for both hole and electron doping.

The conditions under which the $\mathrm{CuO}_{2}$ layers can be doped were discussed by Goodenough et al., see
Refs. 12, 21] for example. Electrons are not easily accepted if the in-plane lattice parameter is too small, $a \lesssim 3.93 \AA$. On the other hand, holes can be introduced if the lattice parameter is not larger than $3.87 \AA$. Strong couplings between the $\mathrm{Cu}-\mathrm{O}$ bond-length fluctuations and the charge carriers, with an anharmonic, multi-well energy landscape and strong charge transfer effects were also predicted in Ref. [12]. This prediction is confirmed by our present study. Nevertheless, both the CAS SCF results on the small clusters and the DMRG estimates for the $p-d$, Hubbard like $\mathrm{CuO}_{4}-\mathrm{CuO}_{4}-\ldots$ chain are rather qualitative. For the CAS SCF calculations, a large part of the dynamic electron correlation effects is not accounted for [4]. Coulomb and (super)exchange interactions beyond the 4-plaquette cluster are also neglected. Within the hole-doped, $p-d$ extended Hubbard model we have neglected "rehybridization" effects [4] arising from the variational optimization of the orbitals. Charge disproportion along the $\mathrm{CuO}$ chain can be induced then only by longer range Coulomb interactions and structural distortions. However, since interactions beyond the nearest neighbors are not explicitly included, longer range Coulomb "correlations" occur through indirect, higher order effects. Each of the two approaches has thus its limitations, but to some extent the two types of methods are complementary. Most importantly, they both predict that the electron-lattice couplings in the doped $\mathrm{CuO}_{2}$ planes are strong and anomalous. The superconductivity might be related to the collective, coherent tunneling of the doped carriers along the multi-well energy landscape. Tunneling effects associated with an electron hole in a double-well potential were analyzed on an oversimplified, hole-doped $\left[\mathrm{CuO}_{2}\right]^{-}$cluster model by Bishop et al., see [11] and references therein. The height of the energy barrier was $400 \mathrm{meV}$ in their model, similar to our results. The amplitude of the O-ion displacements was about two times smaller.

Within the traveling CDW/SDW scenario illustrated schematically in Fig.1, the rigidity of the superconducting condensate is ensured by the inter-carrier Coulomb repulsion and the relatively large AFM interactions between the intervening $\mathrm{Cu} d^{9}$ ions. For a single chain, these couplings are weakened for doping levels below $1 / 3$. In the case of hole doping, it seems that at higher concentrations an homogeneous metallic state is realized, like the $1 / 2$-doped metallic chains in $\mathrm{La}_{1.48} \mathrm{Nd}_{0.40} \mathrm{Sr}_{0.12} \mathrm{CuO}_{4}$ [49]. The charge segregation into $1 / 3$-doped and undoped stripes at optimal doping 12 is supported by inelastic neutron scattering data [50, 51]. Nevertheless, the existence of this type of charge ordering in the $\mathrm{CuO}_{2}$ plane is not definitely established.

We thank A. Yamasaki, G. Stollhoff, G. Kalosakas, and A. T. Filip for fruitful discussions. L. H. acknowledges partial financial support from the Alexander von Humboldt Foundation. 
[1] C.-J. Mei and G. Stollhoff, Phys. Rev. B 43, 3065 (1991).

[2] G. Stollhoff, Phys. Rev. B 58, 9826 (1998).

[3] R. L. Martin, J. Chem. Phys. 98, 8691 (1993).

[4] R. L. Martin, Phys. Rev. B 53, 15501 (1996).

[5] C. J. Calzado, J. F. Sanz, and J. P. Malrieu, J. Chem. Phys. 112, 5158 (2000).

[6] L. Hozoi, S. Nishimoto, and A. Yamasaki, Phys. Rev. B 72, 144510 (2005).

[7] F. C. Zhang and T. M. Rice, Phys. Rev. B 37, 3759 (1988).

[8] Phase Separation in Cuprate Superconductors, Eds. K. A. Müller and G. Benedek (World Scientific, Singapore, 1992).

[9] Phase Separation in Cuprate Superconductors, Eds. E. Sigmund and K. A. Müller (Springer-Verlag, Berlin, 1994).

[10] S. A. Kivelson, I. P. Bindloss, E. Fradkin, V. Oganesyan, J. M. Tranquada, A. Kapitulnik, and C. Howald, Rev. Mod. Phys. 75, 1201 (2003).

[11] A. R. Bishop, D. Mihailovic, and J. Mustre de León, J. Phys.: Condens. Matter 15, L169 (2003).

[12] J. B. Goodenough, J. Phys.: Condens. Matter 15, R257 (2003).

[13] V. Hizhnyakov and E. Sigmund, Physica C 156, 655 (1988).

[14] J. Zaanen and A. M. Oleś, Phys. Rev. B 37, 9423 (1988).

[15] V. J. Emery and G. Reiter, Phys. Rev. B 38, 4547 (1988).

[16] B. I. Kochelaev, J. Sichelschmidt, B. Elschner, W. Lemor, and A. Loidl, Phys. Rev. Lett. 79, 4274 (1997).

[17] L. Proville and S. Aubry, Eur. Phys. J. B 15, 405 (2000); L. Proville and S. Aubry, Eur. Phys. J. B 11, 41 (1999); see also S. Aubry in [8].

[18] A. S. Alexandrov and N. Mott, Polarons and Bipolarons (World Scientific, Singapore, 1995); A. S. Alexandrov, Theory of Superconductivity: From Weak to Strong Coupling (Taylor and Francis, London, 2003).

[19] L. Hozoi, C. Presura, C. de Graaf, and R. Broer, Phys. Rev. B 67, 035117 (2003); L. Hozoi, S. Nishimoto, and A. Yamasaki, Phys. Rev. B 72, 195117 (2005).

[20] For a monograph, see T. Helgaker, P. Jørgensen, and J. Olsen, Molecular Electronic-Structure Theory (Wiley, Chichester, 2000).

[21] M. G. Smith, A. Manthiram, J. Zhou, J. B. Goodenough, and J. T. Markert, Nature 351, 549 (1991).

[22] R. Pou-Amérigo, M. Merchán, I. Nebot-Gil, P.-O. Widmark, and B. O. Roos, Theor. Chim. Acta 92, 149 (1995); P.-O. Widmark, P.-Å. Malmqvist, and B. O. Roos, Theor. Chim. Acta 77, 291 (1990).

[23] D. Muñoz, I. de P. R. Moreira, and F. Illas, Phys. Rev. B 65, 224521 (2002).

[24] C. J. Calzado, J. Cabrero, J. P. Malrieu, and R. Caballol, J. Chem. Phys. 1163985 (2002).

[25] C. de Graaf, Ph. D. thesis, Rijksuniversiteit Groningen (1998).

[26] F. Illas, J. Rubio, J. C. Barthelat, Chem. Phys. Lett. 119, 397 (1985); W. R. Wadt and P. J. Hay, J. Chem. Phys. 82, 284 (1985).

[27] Molcas 6, Department of Theoretical Chemistry, University of Lund, Sweden.

[28] B. H. Botch, T. H. Dunning, and J. F. Harrison, J. Chem.
Phys. 75, 3466 (1981); C. Froese Fischer, J. Phys. B 10, 1241 (1977).

[29] O. K. Andersen, A. I. Liechtenstein, O. Jepsen, and F. Paulsen, J. Phys. Chem. Solids 56, 1573 (1995).

[30] E. Pavarini, I. Dasgupta, T. Saha-Dasgupta, O. Jepsen, and O. K. Andersen, Phys. Rev. Lett. 87, 047003 (2001).

[31] K. Andersson and B. O. Roos, Chem. Phys. Lett. 191, 507 (1992).

[32] C. de Graaf, C. Sousa, I. de P. R. Moreira, and F. Illas, J. Phys. Chem. A 105, 11371 (2001).

[33] P.-Å. Malmqvist, Int. J. Quantum. Chem. 30, 479 (1986); P.-Å. Malmqvist and B. O. Roos, Chem. Phys. Lett. 155, 189 (1989).

[34] X. F. Sun, Y. Kurita, T. Suzuki, S. Komiya, and Y. Ando, Phys. Rev. Lett. 92, 047001 (2004).

[35] R. J. Cava, A. Santoro, D. W. Johnson, and W. W. Rhodes, Phys. Rev. B 35, 6716 (1987).

[36] Compared to the minimal-CAS results reported in Ref. 6], the present study shows that the tendency towards localization on a single anion is less pronounced for larger active spaces. However, we continue to call these broken-symmetry states in the undistorted structure as $\mathrm{O}_{b}^{l}, \mathrm{O}_{b}^{c}$ or $\mathrm{O}_{b}^{r}$-hole states.

[37] M. D. Towler, N. L. Allan, N. M. Harrison, V. R. Saunders, W. C. Mackrodt, and E. Aprá, Phys. Rev. B 50, 5041 (1994).

[38] E. Ruiz, S. Alvarez, P. Alemany, and R. A. Evarestov, Phys. Rev. B 56, 7189 (1997).

[39] Geometry optimizations at a correlated, post-HF level are not possible yet.

[40] A. K. McMahan, R. M. Martin, and S. Satpathy, Phys. Rev. B 38, 6650 (1988); M. S. Hybertsen, M. Schlüter, and N. E. Christensen, Phys. Rev. B 39, 9028 (1989).

[41] For a review, see U. Schollwöck, Rev. Mod. Phys. 77, 259 (2005).

[42] W. A. Harrison, Electronic Structure and the Properties of Solids (Dover, New York, 1989).

[43] See for example [4, 45, 46, 47] and references therein.

[44] K. J. von Szczepanski and K. W. Becker, Z. Phys. B 89, 327 (1992).

[45] O. Rösch and O. Gunnarsson, Phys. Rev. Lett. 92, 146403 (2004).

[46] S. Ishihara and N. Nagaosa, Phys. Rev. B 69, 144520 (2004).

[47] P. Piekarz and T. Egami, Phys. Rev. B 72, 054530 (2005).

[48] More sophisticated methods, e.g., multi-reference CI or multiconfiguration perturbation theory, are needed for treating dynamical correlation effects [20]. Such calculations are beyond the scope of this paper.

[49] J. M. Tranquada, B. J. Sternlieb, J. D. Axe, Y. Nakamura, and S. Uchida, Nature 375, 561 (1995).

[50] R. J. McQueeney, Y. Petrov, T. Egami, M. Yethiraj, G. Shirane, and Y. Endoh, Phys. Rev. Lett. 82, 628 (1999).

[51] T. Egami, Y. Petrov, and D. Louca, J. Supercond.: Incorp. Novel Magn. 13, 709 (2000); T. Egami, J.-H. Chung, R. J. McQueeney, M. Yethiraj, H. A. Mook, C. Frost, Y. Petrov, F. Dogan, Y. Inamura, M. Arai, S. Tajima, and Y. Endoh, Physica B 316-317, 62 (2002). 